\title{
El colegio y los factores de riesgo familiar en la asistencia a programas de prevención familiar del consumo de drogas
}

\author{
The school and family risk factors in attendance at \\ family-based programmes for the prevention of drug use
}

Susana Al-Halabí Díaz*; José Manuel Errasti Pérez ${ }^{\star *}$; José Ramón Fernández Hermida*; José Luis Carballo Crespo**; Roberto Secades VILLA*; Olaya García RodríGUez ${ }^{\star \star \star *}$
${ }^{*}$ Centro de Investigación Biomédica en Red de Salud Mental, CIBERSAM. Área de Psiquiatría - Universidad de Oviedo.

**Departamento de Psicología. Grupo de Conductas Adictivas. Universidad de Oviedo.

***Departamento de Psicología de la Salud. Universidad Miguel Hernández

${ }^{* * * \star D}$ Departamento de Personalidad, Evaluación y Tratamientos Psicológicos. Universidad de Barcelona.

Enviar correspondencia a:

Susana Al-Halabí Díaz. Facultad de Psicología. Plaza Feijoo s/n. 33003 Oviedo - Asturias. E-mail: alsusana@uniovi.es

\section{RESUMEN}

Varios estudios señalan que ciertos aspectos de los centros escolares pueden influir en la decisión de los padres de participar en intervenciones preventivas relacionadas con la salud de sus hijos. Objetivos: 1) Estudiar la relación entre la valoración parental de las convocatorias remitidas desde el centro escolar y los factores de riesgo familiar. 2) Estudiar la existencia de diferencias en la asistencia y abandono de los padres de un programa de prevención familiar del consumo de drogas en función de su valoración de las convocatorias remitidas desde el centro escolar. Muestra: 339 familias de alumnos de $1^{\circ}$ y $2^{\circ}$ de ESO procedentes de 3 I.E.S. de Asturias. Resultados: cabe destacar que los padres con mayor formación académica tienen mejores prácticas parentales $y$, por lo tanto, menores factores de riesgo para el consumo de drogas de sus hijos. Así mismo, la presencia de menos factores de riesgo correlaciona con los items relacionados con la asistencia a las convocatorias del centro escolar y el interés de tales convocatorias, lo que indica que los chicos menos consumidores son aquéllos cuyos padres están más involucrados en su educación escolar. Respecto del efecto de las convocatorias sobre la asistencia y abandono del programa, encontramos que los padres que acudieron a varias sesiones han tenido experiencias previas y satisfactorias con algún tipo de intervenciones preventivas y reciben con gran interés las convocatorias provenientes del centro escolar.

Palabras clave: adolescentes, drogas, participación familiar, prevención familiar, centro escolar, factores de riesgo familiar.

\section{ABSTRACT}

Several studies have indicated that certain aspects of schools may influence parents' decision to participate in preventive interventions related to the health of their children. Objectives: 1) To study the relationship between parental rating of calls for participation sent from the school and family risk factors. 2) To study differences in parents' attendance and drop-out for a family-based drug-use prevention programme according to their rating of the calls for participation sent out by the school. Sample: 339 families of children aged 12-14 from 3 state (public) secondary schools in the region of Asturias (Spain). Results and conclusions: It is noteworthy that parents with higher educational level present better parenting practices, and therefore, fewer risk factors in relation to drug use in their children. Furthermore, the presence of fewer risk factors correlated with the items related to attendance and calls for participation and the interest shown in such calls, indicating that the children least likely to use drugs are those whose parents are most actively involved in their education. As regards the effect of the calls for participation on programme attendance and drop-out, we found that parents who attended several sessions have had previous and satisfactory experience of some kind of preventive intervention, and receive with great interest the calls for participation from the school.

Key words: adolescent, drug, family participation, family prevention, schools, family risk factors. 


\section{INTRODUCCIÓN}

\section{El contexto familiar}

U no de los grupos de factores de riesgo para el consumo de drogas en los adolescentes que ha captado con más claridad la atención de los investigadores ha sido el de los factores familiares. Los resultados de múltiples estudios demuestran que determinadas situaciones familiares están asociadas con un mayor probabilidad de consumo de drogas en los adolescentes (Fernández, Secades, Vallejo, Errasti, 2003; Olsson, Coffey, Toumbourou, Bond, Thomas, y Patton, 2003). Como afirma Becoña (2002), el consumo de drogas tiene como base un proceso de socialización en el que influye de forma destacada la familia, ya que es el ambiente en el que se adquieren determinados tipos de creencias, valores y hábitos que condicionarán más adelante la probabilidad de consumo. Uno de los trabajos pioneros que sentaron las bases de esta linea de investigación fue llevado a cabo por Kandel (1982), en el cual ya se apuntaban algunos de los factores familiares más importantes relacionados con el consumo de drogas de los hijos. Posteriormente, múltiples estudios han perfeccionado esos factores y han postulado la existencia de diversas variables vinculadas al estilo educativo de los padres y al funcionamiento cotidiano familiar en relación a la comunicación, el conflicto o los lazos paternofiliales, como factores de riesgo y de protección implicados en el consumo de drogas por parte de los adolescentes (Hawkins, Catalano y Miller, 1992; Moncada, 1997; Muñoz-Rivas y Graña, 2001; Petterson, Hawkins y Catalano, 1992). Así, existe un cierto consenso entre los investigadores (Secades y Fernández, 2002) acerca de la existencia de cinco grandes factores de riesgo familiares relacionados con las pautas de convivencia y relación entre los padres y los hijos: el tipo de estilo educativo parental, la existencia de destacados conflictos familiares, las actitudes parentales hacia el consumo de drogas, la calidad de los lazos afectivos entre los padres y los hijos, y los estilos de comunicación familiar. Una revisión exhaustiva acerca de los factores de riesgo del consumo de drogas puede encontrarse en Becoña (2002) y en Vázquez y Becoña (2000).

Por este motivo, la prevención familiar ha logrado alcanzar un reconocimiento generalizado como un componente necesario de cualquier plan de prevención (Hogue y Liddle, 1999; Kumpfer y Alvarado, 1995, 2003; Kumpfer y Johnson, 2007), pero el entusiasmo por incluir a los padres en las actividades preventivas se ha visto considerablemente empañado por los numerosos obstáculos que se han encontrado en el reclutamiento y por las modestas tasas de éxito recogidas por la mayoría de los programas (Dishion, Kavanagh, Kiesner, 1998; Durlak, 1997). Por lo tanto, comprometer a los padres en intervenciones preventivas familiares se presenta como uno de los mayores retos a los que se enfrenta el campo de la prevención (Kumpfer, 1991; Resnick y Wojcicki, 1991).

Existen datos suficientes para afirmar que, dentro de la prevención familiar universal, los porcentajes de participación que superan el $30 \%$ de la población objetivo pueden ser considerados ya grandes éxitos, ya que es relativamente frecuente encontrarse con tasas más bajas, del 20-25\% o incluso del 5\% (Ferrer, 1998), en trabajos españoles. Además, estos porcentajes no se distribuyen aleatoriamente entre toda la población, sino que los asistentes suelen ser los padres de familias con menor cantidad o intensidad de factores de riesgo (Klitzner, Gruenewald y Bamberger, 1990). Así, se da el destacable caso de que los principales factores de riesgo familiar relacionados con el consumo de drogas en adolescentes son los mismos que los implicados en la falta de asistencia de los padres a estas actividades de prevención (Cohen y Linton, 1995). Recientemente, un estudio conducido por Haggerty, MacKenzie, Skinner, Harachi, y Catalano (2006) determinó que las variables sociodemográficas y los factores de riesgo para el consumo de drogas asociados a la conducta de los padres estuvieron relacionados con bajas tasas de participación en un programa de prevención.

\section{El contexto escolar}

Por otra parte, además del ambiente familiar, es conocida la importancia y la influencia que tiene el centro escolar no sólo en la formación académica sino también en diversos aspectos del desarrollo durante el periodo vital que abarca desde la infancia hasta la adolescencia. Esta influencia se ejerce tanto de forma directa a través de los compañeros, los profesores, etc., como de manera indirecta, a través de las actitudes y la implicación que los padres tienen hacia el lugar donde sus hijos cursan sus estudios académicos. A este respecto, se ha demostrado en numerosos estudios que una disciplina parental ineficaz acompañada de fracaso académico en los hijos y de la presión ejercida por los compañeros constituyen importantes predictores de conductas antisociales en los adolescentes (Dishion, Patterson, Stoolmiller, Skinner, 1991). Así mismo, la existencia de escasas habilidades sociales o la transición de la educación primaria a la secundaria también son importantes factores de riesgo para la implicación en conductas antisociales (Patterson y Dishion, 1985; Dishion, 1990; Chilcoat, Dishion, Anthony, 1995; Dishion, Capaldi, Spracklen, Li, 1995)

Otros estudios citan algunos aspectos que suelen ir asociados a la participación e implicación de los padres en las actividades del colegio de sus hijos, que incluyen mayor rendimiento académico, actitudes positivas hacia el estudio, menor absentismo escolar, aumento del tiempo que los estudiantes pasan con sus padres y mejores niveles académicos (Epstein, 1991; Greenwood y Hickman, 1991; Marcon, 1999; Shepard y Carlson, 2003). Además de estas ventajas de carácter académico para los hijos, algunos autores han visto el contexto escolar como una alternativa interesante para la prevención y la intervención en problemas familiares (Sáenz Rojas, 2001). Incluso en el ámbito de la salud mental se ha considerado la posibilidad de ofrecer servicios de prevención en diversos contextos que fueran fácilmente accesibles para las familias, como el colegio, para que el mayor número de padres y niños pueda beneficiarse de estos servicios, ya que de otra manera suelen registrarse bajas tasas de participación en los tratamientos de salud mental (Nix, Pinderhughes, Bierman y Maples, 2005). La meta última consistiría en proporcionar desde el colegio una oportunidad para promover cambios positivos en la conducta de los padres y de los niños (Jacob y Doherty, 2006). 


\section{Los programas de prevención familiar en el aula}

No existen muchos estudios que ahonden en la relación entre el centro escolar y la asistencia de los padres a las intervenciones preventivas, pero los que se han publicado a este respecto señalan que ciertos aspectos de los centros escolares pueden influir en la decisión de los padres de participar en los programas de prevención. Algunas de esas características hacen referencia a las creencias de los padres y sus actitudes hacia el colegio, la percepción paterna acerca de la información que reciben sobre sus hijos o la calidad de la instrucción académica (Griffith, 1998; Post, Galanti, Gilijam, 2003). Esta relación entre las actitudes de los padres hacia el centro escolar y su implicación en conductas relacionadas con la salud se ha estudiado en otro tipo de actividades relacionadas con la prevención de comportamientos sexuales de riesgo (Kirby, 2002) o la prevención de la hepatitis $b$ mediante la administración de vacunas (Seid, Simmes, Linton, Leah, Edwards, y Peddecord, 2001).

Si nos centramos de manera precisa en el estudio de la asistencia a los programas de prevención del consumo de drogas, encontramos que el hecho de llevar a cabo estas intervenciones en los colegios goza de varias ventajas añadidas: (1) permite disponer de la información necesaria para establecer contacto con las familias, - teléfono, dirección postal, etc. -, (2) proporciona referencias directas de algún alumno que tenga problemas académicos 0 conductuales, y (3) goza de cierto nivel de credibilidad para la mayoría de los padres. Una investigación realizada por Al-Halabí, Secades, Errasti, Fernández, García y Carballo (2006) sugiere que la credibilidad percibida por parte de los padres de la entidad que convoca a un programa de prevención, - en este caso el centro escolar -, puede ser una variable determinante de la asistencia de los padres a los programas de prevención familiar. También Hahn, Simpson y Kidd (1996) señalaron la importancia de las actitudes del equipo directivo y del personal del centro escolar hacia los padres en la decisión de los padres de participar cuando el programa se desarrolla en este contexto. Así, la calidad de la relación de los padres con los tutores, los profesores y otras figuras dentro de la escuela puede influir en su disposición de participar o no en el programa de prevención ofertado.

En España, tal y como afirma Matellanes (2002), existe cierto consenso acerca de que el medio educativo es el espacio ideal de intervención cuando hablamos de prevención universal. De hecho, se trata del contexto en el que se ha desarrollado la mayor parte de las intervenciones preventivas, aunque no necesariamente de carácter familiar. Sin embargo, las actividades de prevención dirigidas a la familia tienen mayor repercusión y mayores efectos a largo plazo que las estrategias que se centran en los padres solamente o en los hijos solamente, por lo que resulta evidente la necesidad de involucrar a los padres en las actividades y programas que se desarrollen en el ámbito escolar.

\section{OBJETIVO}

De acuerdo con esto, el objetivo de la investigación consistió en estudiar lo siguiente:

- La relación entre la valoración parental de las convocatorias remitidas desde el centro escolar y los factores de riesgo familiar más citados en la literatura, - conflicto familiar, estilo educativo parental, actitudes parentales hacia el consumo de drogas, lazos entre padres e hijos, comunicación familiar y consumo de drogas de los adolescente -

- La existencia de diferencias en la asistencia y abandono de los padres de un programa de prevención familiar del consumo de drogas en función de su valoración de las convocatorias remitidas desde el centro escolar.

- La existencia de diferencias en la asistencia y abandono de los padres de un programa de prevención familiar del consumo de drogas en función de la presencia de factores de riesgo familiar.

\section{MÉTODO}

\section{Participantes}

Esta investigación se realizó con una muestra de 339 familias, - padres e hijos -, procedentes de tres Institutos de Enseñanza Secundaria públicos situados en tres localidades diferentes de la provincia de Asturias, -Cudillero, Gijón y Pola de Siero- que adolecen de problemas relacionados con el consumo de drogas entre los más jóvenes, bien porque constituyen destacadas zonas de ocio vinculado al fenómeno del "botellón", bien porque existe una disponibilidad importante de drogas ilegales en sus alrededores. El perfil socioeconómico de los alumnos asistentes a los centros oscilaba entre medio-bajo y medio. Los padres de los alumnos de Cudillero y Pola de Siero eran mayoritariamente trabajadores del sector primario y entre los padres de los alumnos de Gijón se encontraban por igual trabajadores de los tres sectores. Se trabajó con los cursos de $1^{\circ}$ y $2^{\circ}$ de ESO (12-14 años) en los IES 1 y 2, ya que abarca el rango de edad que aconseja la actividad preventiva que fue llevada a cabo en este estudio. Tan sólo en el IES 3 se trabajó con $1^{\circ}$ E.S.O por limitaciones ajenas a este trabajo. No se registraron diferencias significativas entre las familias de los diferentes centros en los factores de riesgo familiares ni en el consumo de drogas de los alumnos de forma previa a la intervención.

En la Tabla 1 se puede observar la distribución de la muestra que participó en esta investigación. 
Tabla 1. Distribución de los participantes en el estudio.

Número de alumnos

I.E.S

\begin{tabular}{llll}
\hline 1 & 30 & 44 & 74 \\
\hline 2 & 114 & 84 & 198 \\
\hline 3 & 67 & - & 67 \\
\hline Total & 211 & 128 & 339 \\
\hline
\end{tabular}

\section{Variables}

Las variables consideradas en este estudio fueron las siguientes:

1. Factores de riesgo familiar, - (1) conflicto familiar, (2) estilo educativo parental, (3) actitudes parentales hacia el consumo de drogas, (4) lazos entre padres e hijos y (5) comunicación familiar -, y Consumo de Drogas de los adolescentes.

2. Valoración parental de las convocatorias remitidas por el centro escolar medidas a través de los ítems de una encuesta elaborada al efecto.

3. Asistencia al programa de prevención familiar y Abandono de tal programa (asistencia inferior a 5 sesiones principales de las 7 que incluye el programa).

\section{Instrumentos}

\section{Cuestionario de Factores de Riesgo Familiares y Consumo (CFRFC)}

Está compuesto por 47 ítems y se aplicó a los alumnos de la muestra. Para medir las variables del funcionamiento familiar que actúan como factores de riesgo implicados en el consumo de drogas señaladas en el apartado anterior, el cuestionario recoge cuatro escalas del conjunto de Core Measures del Centre for Substance Abuse Prevention (CSAP) norteamericano, - Family Conflict; Poor Family Managment; Parental Attitudes Favorable Toward Drug Use; Family Attachment Scale (Arthur, Hawkins, Catalano y Pollard, 1999) -, y los ítems del cuestionario utilizado por Luengo, Villar, Gómez-Fraguela y Romero (2003). Para medir el consumo de drogas de los alumnos se incluyeron 10 items finales extraídos de la encuesta que el Plan Nacional sobre Drogas (PNSD) utiliza para medir la historia de consumo de drogas de los alumnos españoles (http://www.pnsd.msc.es).

\section{Encuesta de Variables Relacionadas con la Asistencia (EVRA)}

Fue elaborado ad hoc para esta investigación. Está compuesto por 10 ítems y se aplicó a los padres de los alumnos para conocer algunos aspectos de su implicación en el centro escolar, tales como su opinión acerca de la eficacia de la dirección del centro, el número de convocatorias a las que asisten y su valoración en cuanto a la utilidad y el interés de dichas convocatorias (ítems $6,7,8,9$ y 10). También incluye cinco ítems destinados a medir variables sociodemográficas relacionadas con la asistencia (nivel económico, número de hijos y nivel educativo), el uso pasado de recursos preventivos y la satisfacción obtenida de tales recursos (ítems 1, 2, 3, 4, 5). El cuestionario no pretende obtener una puntuación global sobre una única variable, sino que recoge diferentes ítems que serán analizados independientemente. La Tabla 2 recoge este cuestionario.

Tabla 2. Cuestionario de variables relacionadas con la asistencia (EVRA).

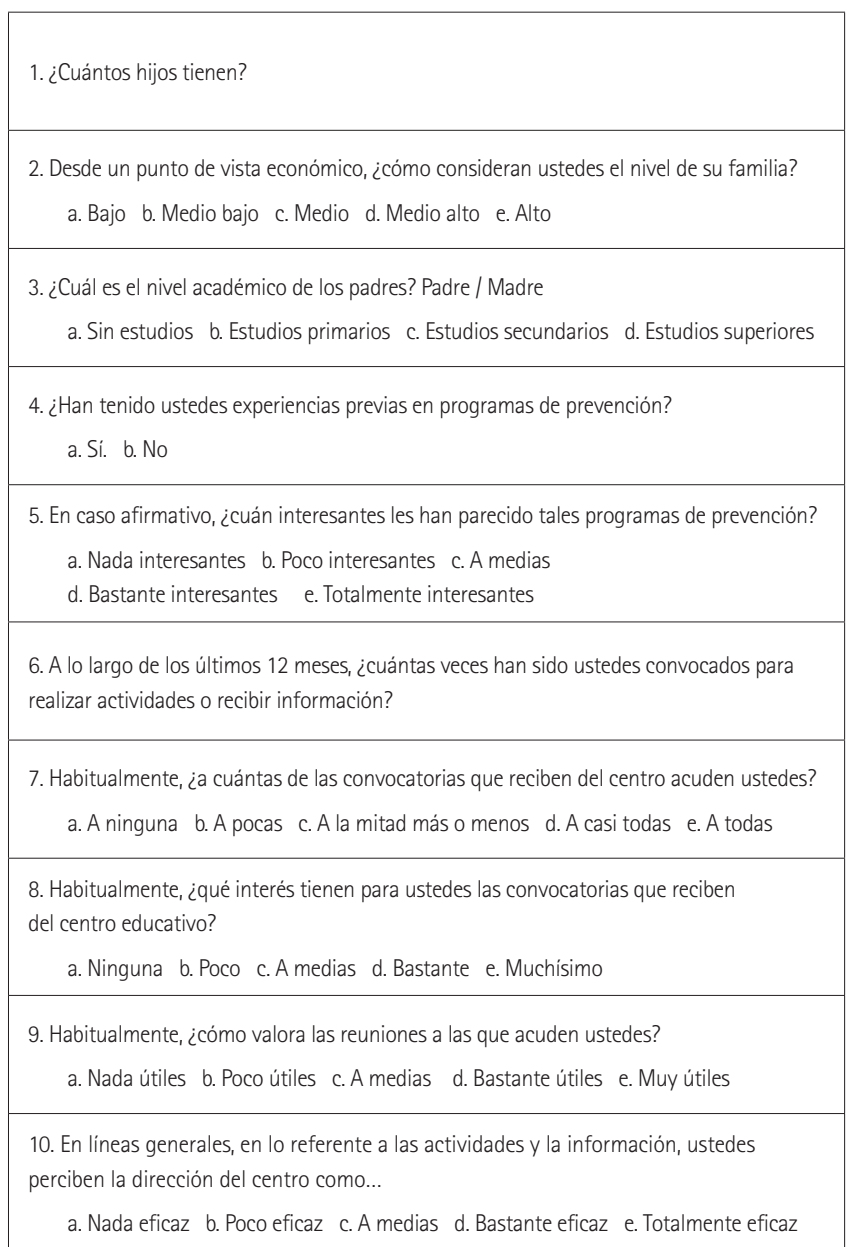




\section{Procedimiento}

Para la aplicación del programa de prevención se seleccionaron inicialmente una serie de centros que cumplian con las características adecuadas, - volumen de alumnado, nivel socioeconómico de los mismos, etcPuestos en contacto con los centros, y en función de la disponibilidad y la colaboración mostrada por los mismos, se eligieron I.E.S. situados en Cudillero, Gijón, Pola de Siero y Oviedo para realizar el estudio. Posteriormente, se acordó con la dirección del centro la aplicación de los cuestionarios, el envío a los padres de la convocatoria al programa de prevención familiar del consumo de drogas y las fechas en las que se llevaría a cabo tal actividad. El programa de prevención aplicado fue "Familias que Funcionan". Este programa es la adaptación española del "Strengthening Families Program 10-14" (SFP 10-14). Consta de 6 sesiones principales, que se aplican consecutivamente una vez a la semana, y 4 sesiones de mantenimiento, que se aplican entre 2 y 6 meses después de finalizar las sesiones principales. Cada sesión dura 2 horas: durante la primera hora los padres se reúnen por un lado con un monitor, y al mismo tiempo los chicos se agrupan por otro lado con su propio monitor; la segunda hora la pasan juntos padres e hijos.

En primer lugar, se aplicó el CFRF a los alumnos de la muestra durante el horario escolar. Posteriormente, se envió a los padres la convocatoria al programa "Familias que Funcionan" remitida desde el centro escolar. La aplicación de los cuestionarios a los padres se llevó a cabo con motivo de la reunión inicial que los centros educativos mantienen con los padres al comienzo del curso que, en este caso, tuvo lugar entre la recepción de la convocatoria y el inicio de "Familias que Funcionan".

Posteriormente comenzaron a aplicarse las sesiones principales del programa "Familias que Funcionan" en los tres institutos seleccionados y con todas las familias, - padres y alumnos -, que acudieron al lugar y a la hora prevista para tal fin. En todos los casos, al finalizar cada una de las sesiones, se pedía a los padres que rellenaran una ficha anónima de asistencia al programa basada en claves. Dos meses más tarde se entregó a los alumnos que habian participado en el programa una convocatoria a las sesiones de mantenimiento remitida por el centro escolar y se llevó a cabo el mismo procedimiento.

A medida que este proceso se estaba realizando, se procedió al registro de los datos para su análisis estadístico mediante el programa estadístico SPSS 14.0

\section{RESULTADOS}

El porcentaje de asistencia de las familias a alguna sesión del programa fue del 8\%. El criterio que se estableció para considerar que una familia no había abandonado la intervención fue la asistencia a 5 o más sesiones principales. Así, dentro de las familias asistentes, el porcentaje de familias que mantuvo una adherencia adecuada al programa fue del $60.7 \%$, mientras que un $39.3 \%$ de las familias abandonó la intervención.
Por otro lado, se realizaron análisis de diferencias de medias (ANOVA de un factor) para estudiar si existían diferencias previas entre los tres I.E.S. en las puntuaciones de los alumnos en las escalas del CFRFC que median factores de riesgo familiares y en la escala que medía el consumo de drogas de los adolescentes. No se registraron diferencias significativas. El consumo de drogas registrado entre los alumnos se centró, como era de esperar, en el alcohol (en los últimos 30 días un 14,1\% lo había consumido de 1 a 2 días, un $2,4 \%$ lo había consumido de 3 a 5 días y un $2 \%$ lo había consumido de 6 a 9 días) y el tabaco (en los últimos 30 días un 3\% lo había consumido de 1 a 2 días, un 1,2\% lo había consumido de 3 a 5 días y un 1\% lo había consumido de 10 a 19 días). Los restantes datos de consumo fueron espurios.

\section{Análisis descriptivo del EVRA}

Si atendemos a los datos sociodemográficos obtenidos podemos decir que, en general, los padres que respondieron a este cuestionario, - aproximadamente el $40 \%$ de la muestra total -, tienen mayoritariamente dos hijos (60\%) y un nivel económico medio (74\%). El 55\% tiene estudios secundarios o superiores y el $42 \%$ tiene estudios primarios (la distribución de esta variable es prácticamente semejante entre los padres y las madres). Sólo el 19\% ha tenido experiencias previas en programas de prevención; dentro de este porcentaje el $63 \%$ de los padres ha encontrado tales programas bastante interesantes o bien totalmente interesantes (22\%), lo que parece reflejar un grado de satisfacción bastante elevado. En lo referente a las preguntas sobre su valoración de las convocatorias de su centro escolar, la mayoría de los padres manifiesta asistir a todas $(58,9 \%)$ o a casi todas $(34,8 \%)$ las convocatorias. Tales convocatorias suelen resultarles muy interesantes $(37,8 \%)$ o bastante interesantes (56\%), así como bastante útiles $(59,2 \%)$ o muy útiles $(23,2 \%)$. Además la mayoría percibe la dirección de su centro como bastante eficaz $(69,5 \%)$.

\section{Correlaciones entre las escalas del CFRFC y los ítems del EVRA}

En la Tabla 3 se puede apreciar la existencia de las siguientes correlaciones significativas:

- Los padres con mayor formación académica tienen un estilo educativo más apropiado, mejores lazos afectivos con sus hijos y buena comunicación con ellos. Así mismo, sus hijos consumen menos.

- Las madres con mayor formación académica tienen mejor estilo educativo, menor conflicto familiar, actitudes desfavorables hacia el uso de drogas y buena comunicación con sus hijos.

- Los padres que acuden a mayor número de convocatorias del centro escolar tienen un estilo educativo más apropiado y sus hijos consumen menos.

- Los padres con mayor interés en las convocatorias del centro escolar tienen hijos menos consumidores. 
Tabla 3. Correlaciones entre las escalas del CFRFC y los ítems del EVRA.

\begin{tabular}{|c|c|c|c|c|c|c|}
\hline \multirow[b]{2}{*}{ EVRA } & \multicolumn{5}{|c|}{ Factores de Riesgo Familiar } & \multirow[b]{2}{*}{ C.D. } \\
\hline & E.E. & C.F. & A.P. & L.F. & C.F. & \\
\hline Item $3 \mathrm{~A}$ & $-.27^{\prime \prime}$ & & & $-.15^{*}$ & $-.20^{\prime \prime}$ & -.19 \\
\hline Item 3B & $-.30 "$ & $-.20^{\prime \prime}$ & $-.16^{*}$ & & $-.26^{\prime \prime}$ & \\
\hline Item 7 & $-.20^{\circ}$ & & & & & -.17 \\
\hline Item 8 & & & & & & -.17 \\
\hline
\end{tabular}

NOTA. E.E. $=$ Estilo Educativo; C.F. $=$ Conflicto Familiar, A.P. $=$ Actitudes Parentales; LF. $=$ Lazos Familiares; C.F. $=$ Comunicación Familiar; C.D. $=$ Consumo de Drogas ${ }^{*} p<.01 .{ }^{*} p<.05$

\section{Diferencias de medias (Prueba $t$ ) en los ítems del EVRA y las escalas del CFRF en función de la variable de agrupación "Asistencia".}

Con el citado análisis se ha examinado la existencia de diferencias en las puntuaciones medias de los ítems del EVRA y las escalas del CFRF entre las familias que asistieron a alguna sesión del programa "Familias que Funcionan", y aquéllas que no lo hicieron. Tal comparación de medias se realizó en función de variables de agrupación dicotómicas, que se crearon a partir de los valores continuos de la variable dependiente de "Asistencia" (hasta 6 sesiones asistidas, que constituye el número de sesiones principales).

En la Tabla 4, podemos observar los dos ítems del EVRA en cuyas puntuaciones se registraron, repetidamente, diferencias significativas. Así, en la puntuación del ítem 5 ("En caso de tener experiencias previas en programas de prevención, ¿cuán interesantes les han parecido tales programas de prevención?") se encontraron diferencias significativas entre las familias que asistieron a más de dos, de tres, de cuatro y de cinco sesiones del programa "Familias que Funcionan" y aquéllas que no lo hicieron. En el caso del item 8 ("Habitualmente, ¿qué interés tienen para ustedes las convocatorias que reciben del centro?"), las puntuaciones son significativamente diferentes entre las familias que asistieron a más de cero, a más de dos y a más de cuatro sesiones del programa "Familias que Funcionan", y aquéllas que no lo hicieron.

Respecto del análisis del CFRFC, como puede apreciarse en la Tabla 5, destaca especialmente la escala de "Consumo de Drogas", que despunta por su elevada significatividad en todos los análisis realizados. Así mismo, existen diferencias significativas en la puntuación de la escala "Actitudes parentales percibidas hacia el uso juvenil de ATOD" entre las familias que asistieron a más de cero, más de una, más de dos, más de cuatro, más de cinco y más de seis sesiones del programa "Familias que Funcionan", y aquéllas que no lo hicieron. No se registraron diferencias significativas en el resto de los factores de riesgo familiares evaluados.

Tabla 4. Prueba t para los ítems del EVRA en función de la Asistencia a "Familias que Funcionan".

\begin{tabular}{lllll}
\hline & M & $\mathrm{N}$ & $\mathrm{M}(\mathrm{SD})$ & \\
\hline
\end{tabular}

\begin{tabular}{|c|c|c|c|c|c|c|}
\hline \multicolumn{7}{|l|}{ ASISTENCIA } \\
\hline más de 0 & & & & $\begin{array}{c}115 a \\
5 b\end{array}$ & $\begin{array}{l}3.93(.645) \\
4.16(.688)\end{array}$ & -2.163 \\
\hline más de 2 & $\begin{array}{c}22 a \\
5 b\end{array}$ & $\begin{array}{l}3.95(.575) \\
4.60(.548)\end{array}$ & $-2.281^{\circ}$ & $\begin{array}{c}123 a \\
19 b\end{array}$ & $\begin{array}{l}4.25(.697) \\
4.53(.513)\end{array}$ & -2.056 \\
\hline más de 3 & $\begin{array}{c}23 a \\
4 b\end{array}$ & $\begin{array}{l}3.96(.562) \\
4.75(.500)\end{array}$ & $-2.638^{*}$ & & & \\
\hline más de 4 & $\begin{array}{c}23 a \\
4 b\end{array}$ & $\begin{array}{l}3.96(.562) \\
4.75(.500)\end{array}$ & $-2.638^{*}$ & $\begin{array}{c}126 a \\
16 b\end{array}$ & $\begin{array}{l}4.25(.692) \\
4.56(.512)\end{array}$ & -2.170 \\
\hline más de 5 & $\begin{array}{c}23 a \\
4 b\end{array}$ & $\begin{array}{l}3.96(.562) \\
4.75(.500)\end{array}$ & $-2.638^{*}$ & & & \\
\hline
\end{tabular}

NOTA. $\mathrm{Na}=$ NO Asistente. $\mathrm{N} \mathrm{b}=$ asistente; $^{*} \mathrm{p}<.05$ 
Tabla 5. Prueba t para las escalas del CFRFC en función de la Asistencia a "Familias que Funcionan".

CFRFC

\begin{tabular}{|c|c|c|c|c|c|c|}
\hline & $\mathrm{N}$ & M (SD) & $t$ & $\mathrm{~N}$ & M (SD) & $t$ \\
\hline \multicolumn{7}{|l|}{ ASISTENCIA } \\
\hline más de 0 & $\begin{array}{c}305 a \\
27 b\end{array}$ & $\begin{array}{l}10.379(.923) \\
10.111(.320)\end{array}$ & $3.301 "$ & $\begin{array}{c}305 a \\
27 b\end{array}$ & $\begin{array}{l}11.485 \text { (.990) } \\
11.814(.483)\end{array}$ & $-3.025^{\prime \prime}$ \\
\hline más de 1 & $\begin{array}{c}309 a \\
21 b\end{array}$ & $\begin{array}{l}10.378(.916) \\
10.047(.218)\end{array}$ & $4.688^{\circ " \prime}$ & $\begin{array}{c}123_{a} \\
19_{b}\end{array}$ & $\begin{array}{l}11.492(.983) \\
11.809(.511)\end{array}$ & $-2.544^{*}$ \\
\hline más de 2 & $\begin{array}{l}311_{a} \\
19_{b}\end{array}$ & $\begin{array}{l}10.376(.914) \\
10.052(.229)\end{array}$ & $4.380^{\circ " m}$ & $\begin{array}{l}311_{\mathrm{a}} \\
19_{\mathrm{b}}\end{array}$ & $\begin{array}{l}11.495(.980) \\
11.789(.535)\end{array}$ & $-2.184^{*}$ \\
\hline más de 3 & $\begin{array}{c}313_{a} \\
17{ }_{b}\end{array}$ & $\begin{array}{l}10.373(.911) \\
10.058(.242)\end{array}$ & $4.028^{\prime \prime}$ & & & \\
\hline más de 4 & $\begin{array}{l}314_{a} \\
16_{b}\end{array}$ & $\begin{array}{l}10.372(.910) \\
10.062(.250)\end{array}$ & $3.833^{\prime \prime \prime}$ & $\begin{array}{c}316_{a} \\
16_{b}\end{array}$ & $\begin{array}{l}11.493(.980) \\
11.875(.341)\end{array}$ & $-3.751^{\prime \prime}$ \\
\hline más de 5 & $\begin{array}{l}316_{\mathrm{a}} \\
14_{\mathrm{b}}\end{array}$ & $\begin{array}{l}10.370(.907) \\
10.071(.267)\end{array}$ & $3.403^{\mathrm{m}}$ & $\begin{array}{c}318_{\mathrm{a}} \\
14_{\mathrm{b}}\end{array}$ & $\begin{array}{l}11.493(.978) \\
11.928(.267)\end{array}$ & $-4.828^{* \prime}$ \\
\hline más de 6 & $\begin{array}{c}320_{a} \\
10\end{array}$ & $\begin{array}{l}10.368(.903) \\
10.000(.000)\end{array}$ & $7.300^{\mathrm{m}}$ & $\begin{array}{c}322_{a} \\
10_{b}\end{array}$ & $\begin{array}{l}11.496(.973) \\
12.000(.000)\end{array}$ & $-9.269^{\prime \prime}$ \\
\hline
\end{tabular}

NOTA. $\mathrm{Na}=$ NO Asistente. $\mathrm{N} \mathrm{b}=$ asistente $^{*} \mathrm{p}<.05 ;{ }^{* *} \mathrm{p}<.005$

Diferencias de medias (Prueba $t$ ) en los ítems del EVRA y las escalas del CFRF en función de la variable de agrupación "Abandono".

En la Tabla 6 podemos observar que se registraron diferencias estadísticamente significativas en las puntuaciones medias del ítem 5 del EVRA ("En caso de tener experiencias previas en programas de prevención, ¿cuán interesantes les han parecido tales programas de prevención?") entre las familias que cumplieron el criterio de "Abandono" del programa (asistencia inferior a 5 sesiones principales) y aquéllas que mantuvieron una buena adherencia al mismo

Tabla 6. Prueba t para los ítems del EVRA en función del Abandono del programa "Familias que Funcionan".

\section{EVRA - İtem 5}

\begin{tabular}{llll}
\hline & $\mathrm{N}$ & $\mathrm{M}(\mathrm{SD})$ & $\mathrm{t}$ \\
\hline \multirow{2}{*}{ Abandono } & $4_{\mathrm{a}}$ & $4.75(.500)$ & \\
& $4_{\mathrm{b}}$ & $4.00(.000)$ & $3.000^{*}$ \\
\hline
\end{tabular}

NOTA. $\mathrm{Na}=$ NO Asistente. $\mathrm{Nb}=$ asistente; ${ }^{*} \mathrm{p}<.05$

\section{DISCUSIÓN}

Uno de los objetivos del presente estudio consistió en estudiar la relación de la valoración parental de las convocatorias remitidas desde el centro escolar y los factores de riesgo familiar y el consumo de los adolescentes. Respecto de este asunto cabe destacar que, en líneas generales y en la dirección esperada, los padres con mayor formación académica tienen mejores prácticas parentales - mejor comunicación, mejor estilo educativo, etc. - (resultados acordes con los aportados por Brook, Brook, Gordon, Whiteman y Cohen, 1990; Conger, Conger, Elder, Lorenz, Simons y Whitbeck, 1992; Dix, 1991; McLoyd, 1990; Simons, Lorenz, Conger y Wu, 1992), y por lo tanto tienen menores factores de riesgo para el consumo de drogas de sus hijos. Así mismo, la presencia de un estilo educativo apropiado y un menor consumo de los hijos correlaciona con los ítems relacionados con la asistencia a las convocatorias del centro escolar y el interés manifestado por los padres en tales convocatorias, lo que indica que los chicos menos consumidores son aquéllos cuyos padres están más involucrados en su educación escolar, lo que alude al efecto protector de dicha actividad parental, tal y como se encuentra en la literatura a este respecto (Kumpfer, Olds, Alexander, Zucker y Gary, 1998). Además, coincide que la falta de familiaridad con la escuela es uno de los factores implicados en la baja participación de los padres en los programas de prevención que se realizan en el contexto escolar (Cohen y Linton, 1995). 
Por otro lado, los adolescentes de la muestra, que tenían entre 12 y 14 años de edad, no refirieron haber consumido drogas ilegales, ya que su consumo se centró, durante el último mes, en el tabaco, el alcohol y las pastillas para dormir. El porcentaje de chicos que sí probaron las citadas drogas en mayor o menor medida fue del 5\%, el $20 \%$ y el $1,5 \%$, respectivamente. Estos datos de consumo parecen "razonables" si tenemos en cuenta la edad de los alumnos de la muestra. Así, los datos encontrados en este estudio se ajustan a las fases de consumo previas que podrian ser esperables en chavales que aún son preadolescentes respecto de las tendencias de consumo recogidas por el Plan Nacional sobre Drogas en su Encuesta Estatal sobre Uso de Drogas en Estudiantes de Enseñanzas Secundarias (14 - 18 años) de 2006-2007.

Otro de los objetivos de este estudió consistió en estudiar la existencia de diferencias en la asistencia y abandono de los padres de un programa de prevención familiar del consumo de drogas en función de su valoración de las convocatorias remitidas desde el centro escolar y de la presencia de factores de riesgo familiar. Respecto de los análisis realizados en función de la variable de agrupación "Asistencia", se hallaron diferencias significativas en las puntuaciones medias de dos escalas del CFRFC y dos ítems del EVRA. Así, estas familias se diferenciaron significativamente en el consumo de drogas de sus hijos, en las actitudes parentales hacia el uso juvenil de alcohol y otras drogas, en las experiencias previas de prevención y la satisfacción obtenida de ellas y en el interés que les despierta las convocatorias del centro escolar. Los padres que acudieron a varias sesiones del programa tienen actitudes más negativas hacia el consumo de drogas, lo que funciona como factor de protección para el consumo de sus hijos, que de hecho consumen menos. Estos padres también han tenido experiencias previas y satisfactorias con algún tipo de intervenciones preventivas, un factor muy destacado en el modelo de Spoth y Redmond (1995), y reciben con gran interés las convocatorias provenientes del centro escolar, resultado acorde con lo obtenido por Al-Halabí et al. (2006), Griffith (1998) y Post et al. (2003). Parece coherente, por lo tanto, que sean este tipo de padres los que acuden al programa de prevención ya que las experiencias beneficiosas en otro tipo de actividades preventivas $y$ el interés en las actividades del centro escolar parecen aspectos motivantes para participar en el programa.

Por otra parte, dentro de la muestra que asistió al programa, las puntuaciones medias obtenidas en el ítem que aludía a la satisfacción alcanzada en experiencias previas de prevención mostró diferencias significativas entre las familias que cumplieron el criterio de Abandono del programa y aquéllas que mantuvieron una buena adherencia al mismo. Este resultado es coherente con los hallazgos de Spoth y Redmond (1995) que, aunque en su modelo no estudiaron concretamente el tema de la adherencia a un programa, encontraron una fuerte relación predictiva entre el uso pasado de recursos parentales y la disposición para participar en un programa de habilidades parentales.
Los resultados de este tipo de estudios sugieren que los padres manifiestan, en general, una buena disposición para involucrarse en las labores preventivas pero, de hecho, su participación real en las acciones propuestas es muy escasa, así como también lo es su nivel de utilización de recursos comunitarios como las AMPAS. A este respecto, Pinazo y Pons (2002) señalan que los padres, como principales responsables de la educación de sus hijos, tienen una importancia fundamental en todo planteamiento preventivo, por lo que deben asumir un papel activo en la implementación de este tipo de intervenciones. Estos autores indican la necesidad de formar a los padres para que adquieran los conocimientos y competencias requeridas para desempeñar su papel educativo adecuadamente.

Por su parte, Spoth, Goldberg y Redmond (1999) sugieren que los esfuerzos de captación deben apoyarse en la flexibilidad de los programas, enfatizando los beneficios que pueden obtenerse por participar en ellos y asegurando absoluta confidencialidad. En este sentido Calafat y Amengual (1999) mencionan una serie de condiciones necesarias para la participación de los padres:

- Debe informarse a los padres de los proyectos de la escuela acerca de la prevención del consumo de alcohol y otras drogas e invitarles a participar en la definición de los objetivos de dichas actividades.

- Es necesario que los padres perciban esta invitación como auténtica, lo que significa que efectivamente se tendrán en cuenta sus puntos de vista ya que, a veces, los padres tienen la impresión de que sólo son necesarios para cumplir las instrucciones de la escuela, para proporcionar recursos o apoyos a algunas actividades, o para trabajar como voluntarios. Una mayor implicación requiere que se les considere como participantes reales en la educación de sus hijos.

- No se debe concentrar toda la responsabilidad de la educación en los padres, pero tampoco presentar la escuela como el contexto en el que se puede arreglar todo.

- Es necesario tener en cuenta que para muchos padres resulta complicado abordar algunas cuestiones aisladamente en casa, y por lo tanto, la escuela puede apoyarles o suplir ese déficit a través de los programas y las actividades para la educación sobre el consumo de drogas $u$ otras cuestiones de salud.

- Puede ocurrir que el profesorado perciba la participación de los padres como impertinente, y también hay padres que prefieren que la escuela se ocupe de toda la responsabilidad educativa. Por ello, es necesario que ambos, padres y profesores, se convenzan de que la colaboración es beneficiosa para todos. 


\section{Limitaciones del estudio}

El problema que surge con la descripción de la muestra de padres que aquí se presenta es que se basa en los datos aportados por aquellos padres que acudieron a las reuniones iniciales del curso escolar, momento en el que se aplicó el EVRA. Este tipo de reuniones son, sin duda, las más concurridas, pero aun así, cabe suponer que asisten los padres más volcados o preocupados por la educación de sus hijos, o al menos podemos pensar que no acuden aquéllos que quizá tengan más desinterés a este respecto o piensen que este tipo de reunión no les va a aportar información útil o relevante. Sería muy interesante conseguir un mayor número de cuestionarios cumplimentados por los padres mediante correo, e-mail o encuestas telefónicas, especialmente de aquéllos que no suelen acudir a las convocatorias ordinarias que reciben desde el centro escolar. Es posible que esos padres manifestaran una opinión diferente o menos satisfactoria acerca del funcionamiento del equipo directivo del colegio, o tal vez, sencillamente, es posible que tales padres no estén muy implicados en los aspectos escolares de sus hijos o no puedan acudir por motivos diversos.

Por otra parte, existen otras cuestiones importantes que no conviene olvidar a la hora de intervenir con los padres en el ámbito escolar. En primer lugar, en nuestro país la participación de los padres en el funcionamiento cotidiano de los centros es escasa y, por lo tanto, sería deseable que esta participación fuese mayor, aunque eso supondría "sacudir las raíces" de algunos aspectos muy anclados en el sistema educativo y en la percepción de los padres acerca de los colegios. En segundo lugar, en el propio ámbito escolar se cuenta poco con los padres a la hora de implementar y ejecutar los programas. Así, en ocasiones los profesores sienten que se invaden sus competencias y ello se refleja, a su vez, en las tasas de participación de los padres en este tipo de actividades. Por lo tanto, es evidente que las familias deberian adquirir un papel un poco más destacado en los centros escolares, pero también es evidente que esa relación es difícil y que en ocasiones puede convertirse en una fuente de pequeños conflictos entre padres y profesores (Matellanes, 2002).

\section{AGRADECIMIENTOS}

Este trabajo ha sido financiado por el proyecto MSC-05PND-01 del Plan Nacional sobre Drogas.

\section{REFERENCIAS}

Al-Halabi Diaz, S., Secades Villa, R., Errasti Pérez, J. M., Fernández Hermida, J. R., Garcia Rodríguez, 0. y Carballo Crespo, J. L. (2006). Family predictors of parent participation in an adolescent drug abuse prevention program. Drug and Alcohol Review, 25, 323-327.

Arthur, M. W., Hawkins, J. D., Catalano, R. y Pollard, J. A. (1999). Core measures initiative phase I recommendations. Center for Substance Abuse Prevention (CSAP).

Becoña, E. (2002). Bases científicas de la prevención de las drogodependencias. Madrid: Plan Nacional Sobre Drogas.

Brook, J. S., Brook, D. W., Gordon, A. S., Whiteman, M. y Cohen, P. (1990). The psychological etiology of adolescescent drug use: a family interactional approach. Genetic, Social and General Psychology Monographs, 116, 111-267.

Calafat, A., y Amengual, M. (1999). Educación sobre el alcohol. Madrid: Delegación del Gobierno para el Plan Nacional sobre Drogas.

Chilcoat, D., Dishion, T. y Anthony, J. (1995). Parent monitoring and the incidence of drug sampling in urban elementary school children. American Journal of Epidemiology, 141, 25-31

Cohen, D. A. y Linton, K. L. (1995). Parent participation in an adolescent drug abuse prevention program. Journal of Drug Education, 25, 159-169.

Conger, R. D., Conger, K. J., Elder, G. H., Lorenz, F. O., Simons, R. L. y Whitbeck, L. B. (1992). A family process model of economic hardship and adjustment of early adolescent boys. Child Development, 63, 526-541.

Dishion, T. (1990). The family ecology of boy's peer relations in middle childhood. Child Development, 61, 874-892.

Dishion, T., Capaldi, D., Spracklen, K. y Li, F. (1995). Peer ecology of male adolescent drug use. Development and Psychopathology, 7, 803-824.

Dishion, T., Kavanagh, K. y Kiesner, J. (1998). Prevention of early adolescent substance abuse among high-risk youth: a multiple gating approach to parent intervention. En R. Ashery, E. Robertson y K. Kumpfer (Eds.), Drug abuse prevention through family interventions. Rockville. M.D.: NIDA research Monograph 177.

Dishion, T., Patterson, G., Stoolmiller, M., y Skinner, M. (1991). Family, school, and behavioral antecedents to early adolescents involvement with antisocial peers. Developmental Psychology, 27, 172-180.

Dix, T. (1991). The affective organization of parenting: adaptive and maladaptive processes. Psychological Bulletin, 110, 3-25.

Durlak, J. (1997). Successful prevention programs for children and adolescents. New York: Plenum Press.

Epstein, J. L. (1991). Effects on student achievement in teachers' practice of parent involvement. Advances in Reading/Language Research, 5, 261-276.

Fernández Hermida, J. R., Secades Villa, R., Vallejo Seco, G. y Errasti Pérez, J. M. (2003). Evaluation of what parents know about their children's drug use and how they perceive the most common family risk factors. Journal of Drug Education, 33, 334-350

Ferrer, X. (1998). La prevención del abuso de drogas en el ámbito familiar. Comunicación presentada en el Congreso Europeo sobre Prevención de Drogodependencias, Madrid.

Greenwood, G. E. y Hickman, C. W. (1991). Research and practice in parent involvement: implications for teacher education. Elementary School Journal, 91, 279-288.

Griffith, J. (1998). The relation of school structure and social environment to parent involvement in elementary schools. Elementary School Journal, 1, 53-80.

Haggerty, K., MacKenzie, E., Skinner, M., Harachi, T. y Catalano, R. F. (2006). Participation in "Parents Who Care": Predicting program initiation and exposure in two different program formats. Journal of Primary Prevention, 27, 47-65.

Hahn, E., Simpson, M. R. y Kidd, P. (1996). Cues to parent involvement in drug prevention and school activities. Journal of School Health, 66, 165-170. 
Hawkins, J. D., Catalano, R. F. y Miller, J. Y. (1992). Risk and protective factors for alcohol and other drug problems in adolescence and early adulthood: implications for substance abuse prevention. Psychological Bulletin, 112, 64-105.

Hogue, A. y Liddle, H. A. (1999). Family-based preventive intervention: an approach to preventing substance use and antisocia behavior. American Journal of Orthopsychiatry, 69, 278-293.

Jacob, J. y Doherty, W. J. (2006). Healthy family systems. En G. G. Bear, y K. M. Minke (Eds.), Children's needs III: Development, prevention and intervention. Washington, DC, US: National Association of School Psychologist.

Kandel, D. B. (1982). Epidemiological and psychosocial perspectives on adolescents drug use. Journal of American Academic Clinical Psychiatry, 21, 328-347.

Kirby, D. (2002). The impact of schools and school programs upon adolescent sexual behavior. Journal of Sex Research, 39, 27-33.

Klitzner, M., Gruenewald, P. y Bamberger, E. (1990). The assessment of parent-led prevention programs: A preliminary assessment of impact. Journal of Drug Education, 20, 77-94.

Kumpfer, K. L. (1991). How to get hard to reach parents involved in parenting programs. En D. Pines (Ed.), Parent training is prevention: preventing alcohol and other drug problems among youth in the family (pp. 87-95). Washington, D.C.: Government Printing Office: DHHS Publication.

Kumpfer, K. L. y Alvarado, R. (1995). Strengthening families to prevent drug use in multiethnic youth. En G. J. Botvin, S. Schinke, y M. A. Orlandi (Eds.), Drug abuse prevention with multiethnic youth (pp. 255-294). Thousand Oaks, CA: Sage.

Kumpfer, K. L. y Alvarado, R. (2003). Family-strengthening approaches for the prevention of youth problem behaviors. American Psychologist, 58, 457-465.

Kumpfer, K. L. y Johnson, J. L. (2007). Intervenciones de fortalecimiento familiar para la prevención del consumo de sustancias en hijos de padres adictos. Adicciones, 19, 13-25.

Kumpfer, K. L. Olds, D. L., Alexander, J. F., Zucker, R. A., y Gary, L. E. (1998). Family etiology of youth problems. En R. S. Ashery, E. B. Robertson, y K. L. Kumpfer (Eds.), Drug abuse prevention trough family interventions. Rockville, MD: US Department of Health and Human Services.

Luengo Martín, M. A., Villar Torre, P., Gómez Fraguela, J.A. y Romero Triañes, E. (2003). Una propuesta de evaluación de variables familiares en al prevención de la conducta problema en la adolescencia. Psicothema, 15, 581-588.

Marcon, R. A. (1999). Positive relationship between parent school involvement and public school inner-city preschoolers' development and academic performance. School Psychology Review, 28, 395-412.

Matellanes, M. (2002). La prevención de las drogodependencias. ¿Es posible la integración de la intervención comunitaria, escolar y familiar? En J. R. Fernández. Hermida, y R. Secades Villa (Eds.), Intervención familiar en la prevención de las drogodependencias (pp. 171-196). Madrid: Plan Nacional sobre Drogas.

McLoyd, V. C. (1990). The impact of economic hardship on black families and children: Psychological distress, parenting, and socioemotional development. Child Development, 61, 311-346.

Moncada, S. (1997). Factores de riesgo y de protección en el consumo de drogas. En Plan Nacional sobre Drogas (Ed.), Prevención de las drogodependencias. Análisis y propuestas de actuación (pp. 85-101). Madrid: Plan Nacional Sobre Drogas.

Muñoz-Rivas, M. J. y Graña, J. L. (2001). Factores de riesgo y de protección para el consumo de drogas en los adolescentes. Psicothema, 13, 87-94

Nix, R. L., Pinderhughes, E. E., Bierman, K. L. y Maples, J. J. (2005). Decoupling the relation between risk factors for conduct problems and the receipt o intervention services: participation across multiple components of a prevention program. American Journal of Community Psychology, 36, 307-325.

Olsson, C., Coffey, C., Toumbourou, J., Bond, L., Thomas, L. y Patton, G. (2003). Family risk factors for cannabis use: a populationbased survey of Australian secondary school students. Drug and Alcohol Review, 2, 143-152.

Patterson, G. y Dishion, T. (1985). Contributions of families and peers to delinquency. Criminology, 23, 63-79.

Petterson, P. L., Hawkins, J. D. y Catalano, R. (1992). Evaluating comprehensive community drug risk reduction interventions. Design challenges and recommendations. Evaluation Review, 16, 579-602.

Pinazo, S., y Pons, J. (2002). La implicación de los padres en los programas preventivos del consumo de drogas: un estudio empírico. En J. R. Fernández Hermida y R. Secades Villa (Eds.), Intervención familiar en la prevención de las drogodependencias (pp. 325-364). Madrid: Plan Nacional Sobre Drogas.

Plan Nacional Sobre Drogas. Recuperado de http://www.pnsd.msc.es

Post, A., Galanti, M. R. y Gilijam, H. (2003). School and family participation in a longitudinal study of tobacco use: some methodological notes. European Journal of Public Health, 1, 75-76.

Resnick, H., y Wojcicki, M. (1991). Reaching and retaining highrisk youth and their parents in prevention programs. En E. N. Gplerud (Ed.), Preventing adolescent drug use: From theory to practice (pp. 91-126). Rockville, D.C. Office for Substance Abuse Prevention: Prevention Monograph № 8.

Secades Villa, R. y Fernández Hermida, J.R. (2002). Factores de riesgo para el uso de drogas: un estudio empirico español. En Plan Nacional sobre Drogas (Ed.), Intervención familiar en la prevención de las drogodependencias. Madrid: Plan Nacional sobre Drogas.

Seid, M., Simmes, D. R., Linton, L. S., Leah, C. E., Edwards, C. C. y Peddecord, K. M. (2001). Correlates of vaccination for hepatitis $B$ among adolescents: results from a parent survey. Archives of Pediatrics and Adolescent Medicine, 155, 921-926.

Shepard, J. y Carlson, J. (2003). An Empirical Evaluation of SchoolBased Prevention Programs That Involve Parents. Psychology in Schools, 40, 641-656.

Sáen Rojas, M.A. (2001). Evaluación de proceso de un programa de prevención de drogas en la escuela primaria costarricense. Adicciones, 13, 323-334

Simons, R. L., Lorenz, F. O., Conger, R. D. y Wu, C. (1992). Support from spouse as mediator and moderator of the disruptive influence of economic strain on parenting. Child Development, 63, 12821301.

Spoth, R., Goldberg, C., y Redmond, C. (1999). Engaging families in longitudinal preventive intervention research: discrete-time survival analysis of socioeconomic and social-emotional risk factors. Journal of Consulting and Clinical Psychology, 67, 157163.

Spoth, R. y Redmond, C. (1995). Parent motivation to enroll in parenting skills programs: a model of family context and health belief predictors. Journal of Family Psychology, 9, 294-310.

Vázquez, F. y Becoña, E. (2000). Factores de riesgo y escalada cannabinoide. Adicciones, 12 (Supl. 2), 175-184. 